\title{
Clinical Characteristics of Disseminated Strongyloidiasis, Japan, 1975-2017
}

Mitsuru Mukaigawara, Masashi Narita, Soichi Shiiki, Yoshihiro Takayama, Shunichi Takakura, Tomokazu Kishaba

\section{Medscape ACTIVITY}

In support of improving patient care, this activity has been planned and implemented by Medscape, LLC and Emerging Infectious Diseases. Medscape, LLC is jointly accredited by the Accreditation Council for Continuing Medical Education (ACCME), the Accreditation Council for Pharmacy Education (ACPE), and the American Nurses Credentialing Center (ANCC), to provide continuing education for the healthcare team.

Medscape, LLC designates this Journal-based CME activity for a maximum of 1.00 AMA PRA Category 1 Credit(s) ${ }^{\mathrm{TM}}$. Physicians should claim only the credit commensurate with the extent of their participation in the activity.

Successful completion of this CME activity, which includes participation in the evaluation component, enables the participant to earn up to $1.0 \mathrm{MOC}$ points in the American Board of Internal Medicine's (ABIM) Maintenance of Certification (MOC) program. Participants will earn MOC points equivalent to the amount of CME credits claimed for the activity. It is the CME activity provider's responsibility to submit participant completion information to ACCME for the purpose of granting ABIM MOC credit.

All other clinicians completing this activity will be issued a certificate of participation. To participate in this journal CME activity: (1) review the learning objectives and author disclosures; (2) study the education content; (3) take the post-test with a $75 \%$ minimum passing score and complete the evaluation at http://www.medscape.org/journal/eid; and (4) view/print certificate. For CME questions, see page 637 .

Release date: February 19, 2020; Expiration date: February 19, 2021

Learning Objectives

Upon completion of this activity, participants will be able to:

- Describe classification of disseminated strongyloidiasis into 3 clinical phenotypes, according to a case series

- Determine clinical and laboratory findings in disseminated strongyloidiasis, according to a case series

- Identify treatment of disseminated strongyloidiasis, according to a case series

\section{CME Editor}

Jude Rutledge, BA, Technical Writer/Editor, Emerging Infectious Diseases. Disclosure: Jude Rutledge has disclosed no relevant financial relationships.

\section{CME Author}

Laurie Barclay, MD, freelance writer and reviewer, Medscape, LLC. Disclosure: Laurie Barclay, MD, has disclosed no relevant financial relationships.

\section{Authors}

Disclosures: Mitsuru Mukaigawara, MD; Masashi Narita, MD; Soichi Shiiki, MD; Yoshihiro Takayama, MD; Shunichi Takakura, MD; and Tomokazu Kishaba, MD, have disclosed no relevant financial relationships.

Clinical characteristics of disseminated strongyloidiasis, the severest form of strongyloidiasis, are not well described. We conducted a retrospective, consecutive chart review of patients with disseminated strongyloidiasis admitted to Okinawa Chubu Hospital in Okinawa, Japan, during January 1975-December 2017. The 70 patients were classified into 3 clinical phenotypes: dissemination (32 patients [45.7\%]), occult dissemination with meningitis caused by enteric organisms (12 patients [17.1\%]), and occult dissemination with culture-negative suppurative meningitis (26 patients
[37.1\%]). Associated mortality rates were 56.3\%, $16.7 \%$, and $11.5 \%$, respectively, and sepsis occurred in $40.6 \%$, $58.3 \%$, and $11.5 \%$ of cases, respectively. Common symptoms included fever (52.9\% of patients), headache $(32.9 \%)$, and altered mental status $(24.3 \%)$. Patients were treated with thiabendazole (before 2003) or ivermectin (after 2003). Our findings show that disseminated strongyloidiasis has clinical phenotypes in terms of severity and that identification of occult dissemination, a mild form with prominent neurologic manifestations, is lifesaving.

Author affiliations: Harvard Kennedy School, Cambridge, Massachusetts, USA (M. Mukaigawara); Okinawa Chubu Hospital, Uruma, Okinawa, Japan (M. Mukaigawara, M. Narita, S. Shiiki, Y. Takayama, S. Takakura, T. Kishaba) 
$S^{\text {t }}$ trongyloidiasis is a nematode infection caused by Strongyloides stercoralis, a parasitic roundworm. Parasites of the genus $S$. stercoralis are characteristic for their ability to replicate within hosts (1). Patients have clinical characteristics of systemic strongyloidiasis when their immune status is impaired (2), such as through the use of steroids (3), medication associated with organ transplantation (4), and initiation of chemotherapy (5). Strongyloidiasis has been prevalent in the tropics and subtropics and is reemerging as a critical disease in the immunocompromised patient population (6).

The life cycle of the $S$. stercoralis roundworm consists of external and autoinfective cycles (7). Infective filariform larvae penetrate the skin, enter the venous circulation, and migrate to the pulmonary circulation. The larvae are then swallowed and remain in the small intestine to mature into the parthenogenic female adult $S$. stercoralis worm (8). The noninfectious rhabditiform offspring can develop into infective filariform larvae while still in the intestine and reinfect the host. These autoinfective filariform larvae enable the unique autoinfective life cycle of $S$. stercoralis parasites to continue indefinitely in humans.

When the host immune status is compromised, the autoinfective cycle can be enhanced, enabling increasing numbers of autoinfective larvae to disseminate throughout the body. The clinical complications of disseminated strongyloidiasis are a result of the increasing numbers of autoinfective larvae traveling through the body, with many larvae carrying enteric bacteria from the intestine (9). Penetrated larvae carry enteric organisms to multiple organ systems, causing sepsis $(10-12)$, pneumonia $(10,11,13)$, and meningitis $(10,11,14-18)$.

Early recognition of dissemination is essential, yet little has been known about its clinical, laboratory, and microbiological characteristics (1). Our study aimed to provide characteristics of patients with disseminated strongyloidiasis. We conducted a retrospective, consecutive chart review of patients diagnosed with disseminated strongyloidiasis in an area of human T-cell lymphotrophic virus type 1 (HTLV1) infection endemicity in Japan during January 1975December 2017.

\section{Methods}

\section{Study Setting}

This study was conducted at Okinawa Chubu Hospital in Okinawa, Japan, which has a subtropical climate; strongyloidiasis is endemic to Okinawa (19). The region also has a high prevalence of HTLV-1 infection (20). Okinawa Chubu Hospital is one of the largest teaching hospitals in Okinawa.

\section{Study Design and Patients}

We consecutively reviewed the charts of adult patients ( $\geq 18$ years of age) diagnosed with disseminated strongyloidiasis during January 1975-December 2017 and admitted to Okinawa Chubu Hospital. We classified disseminated strongyloidiasis into 3 clinical phenotypes: dissemination, occult dissemination with meningitis caused by enteric organisms (14), and occult dissemination with culture-negative suppurative meningitis (15).

We defined dissemination as filariform larvae detected from specimens other than the gastrointestinal tract (i.e., feces, duodenal fluid, and gastric juice). We defined occult dissemination with meningitis caused by enteric organisms as meningitis caused by enteric organisms combined rhabditiform larvae detected from the gastrointestinal tract (e.g., in feces and gastric juice); enteric organisms were Bacteroides spp., Enterococcus spp., Escherichia coli, Enterobacter spp., Klebsiella spp., Bifidobacterium spp., Clostridium perfringens, Proteus mirabilis, Streptococcus gallolyticus (bovis group), and Campylobacter spp. (21). We defined occult dissemination with culture-negative suppurative meningitis as cerebrospinal fluid (CSF) culture-negative meningitis with CSF neutrophil-dominant pleocytosis of $>500$ cells / $\mathrm{mm}^{3}(16)$ combined with rhabditiform larvae detected from the gastrointestinal tract (e.g. in feces and gastric juice).

Patients with nosocomial meningitis and meningitis patients with prior receipt of antibiotics (within 7 days of performing lumbar puncture) were excluded from the analysis. We performed parasitologic examinations by direct microscopic examinations, formalin-ether concentration technique, or both interchangeably on the basis of clinical symptoms.

\section{Ethics Statement}

We collected demographic data, clinical characteristics, clinical diagnosis, and microbiologic data for all patients in a secure database after identifying information of included patients was removed. This study was approved by the Institutional Review Board of Okinawa Chubu Hospital (approval no. H29-75).

\section{Results}

\section{Patient Population}

We identified 70 patients during the study period (Table 1); 42 (60\%) were men. Median age of patients 
Table 1. Demographic characteristics of patients with disseminated strongyloidiasis, Okinawa Chubu Hospital, Uruma, Japan, 1975-2017*

\begin{tabular}{|c|c|c|c|c|}
\hline Characteristic & All patients & Dissemination & $\begin{array}{c}\text { Occult dissemination } \\
\text { with meningitis/enteric } \\
\text { organisms }\end{array}$ & $\begin{array}{c}\text { Occult dissemination } \\
\text { with culture-negative } \\
\text { suppurative meningitis }\end{array}$ \\
\hline No. patients & $70 / 70(100)$ & $32 / 70(45.7)$ & $12 / 70(17.1)$ & $26 / 70(37.1)$ \\
\hline \multicolumn{5}{|l|}{ Sex } \\
\hline M & $42(60)$ & $19(59.4)$ & $6(50)$ & $17(65.3)$ \\
\hline $\mathrm{F}$ & $28(40)$ & $13(40.6)$ & $6(50)$ & $9(34.7)$ \\
\hline Median age, y (range) & $61(21-96)$ & $67(25-96)$ & $47(21-84)$ & $57(38-92)$ \\
\hline \multicolumn{5}{|l|}{ Immunocompromised status } \\
\hline HTLV-1 infection† & $36 / 40(90.0)$ & $17 / 20(85.0)$ & $8 / 9(88.9)$ & $11 / 11(100)$ \\
\hline Steroid use & $6(8.6)$ & $5(15.6)$ & $1(8.3)$ & $0(0)$ \\
\hline Solid organ malignancy & $6(8.6)$ & $4(12.5)$ & $2(16.7)$ & $0(0)$ \\
\hline Diabetes mellitus & $4(5.7)$ & $4(12.5)$ & $0(0)$ & $0(0)$ \\
\hline Cirrhosis & $2(2.9)$ & $2(6.3)$ & $0(0)$ & $0(0)$ \\
\hline Death & $23(32.9)$ & $18(56.3)$ & $2(16.7)$ & $3(11.5)$ \\
\hline
\end{tabular}

was 61 years (range 21-96 years); all patients were born before 1964. Thirty-two patients (32/70 [45.7\%]) had dissemination, 12 (12/70 [17.1\%]) had occult dissemination with meningitis caused by enteric organisms, and 26 (26/70 [37.1\%]) had occult dissemination with culture-negative suppurative meningitis. Thirty-six tested patients (36/39 [92.3\%]) had positive serologic assay results for HTLV-1. Six patients (6/70 [8.6\%]) had solid organ malignancy, 5.7\% $(4 / 70)$ had diabetes mellitus, and $2.9 \%(2 / 70)$ had cirrhosis. Six patients $(8.6 \%)$ regularly used steroids; doses were equivalent to 5-30 $\mathrm{mg}$ of daily prednisolone. The mortality rate was highest in the dissemination group (18/32 [56.3\%]), followed by the occult dissemination with meningitis caused by enteric organisms (2/12 [16.7\%]) group and the occult dissemination with culture-negative suppurative meningitis $(3 / 26[11.5 \%])$ group.

The number of annually reported cases was decreasing during the study period. The maximum number of annual cases was 7 (Figure). Most strongyloidiasis cases in the last decade of the study period were classified as dissemination.

\section{Clinical Manifestations}

Common symptoms included fever (37/70 [52.9\%] patients), headache $(23 / 70[32.9 \%])$, altered mental status (17/70 [24.3\%]), and nausea/vomiting (10/70 [14.3\%]) (Table 2). In terms of laboratory findings, the average absolute eosinophil count was 225 cells/ $\mu \mathrm{L}$ (range $0-1,482$ cells $/ \mu \mathrm{L}$ ).

The main clinical diagnoses included meningitis, pneumonia, sepsis, and paralytic ileus (Table 2). Twenty-three patients $(32.9 \%)$ had clinical manifestations of sepsis caused by enteric organisms, 22 patients $(31.4 \%)$ had clinical manifestations of meningitis caused by enteric organisms, and 34 patients (48.6\%) had clinical manifestations of culture-negative suppurative meningitis. Nine patients had highly critical conditions with evidence of multiorgan involvement, including the gastrointestinal (paralytic ileus), pulmonary (pneumonia caused by enteric organisms), central nervous (meningitis), and circulatory (sepsis caused by enteric organisms) systems. The mortality rate was $77.8 \%(7 / 9)$ in these patients. Such clinical features (22) have been referred to as "full-blown" dissemination at our institution (10).

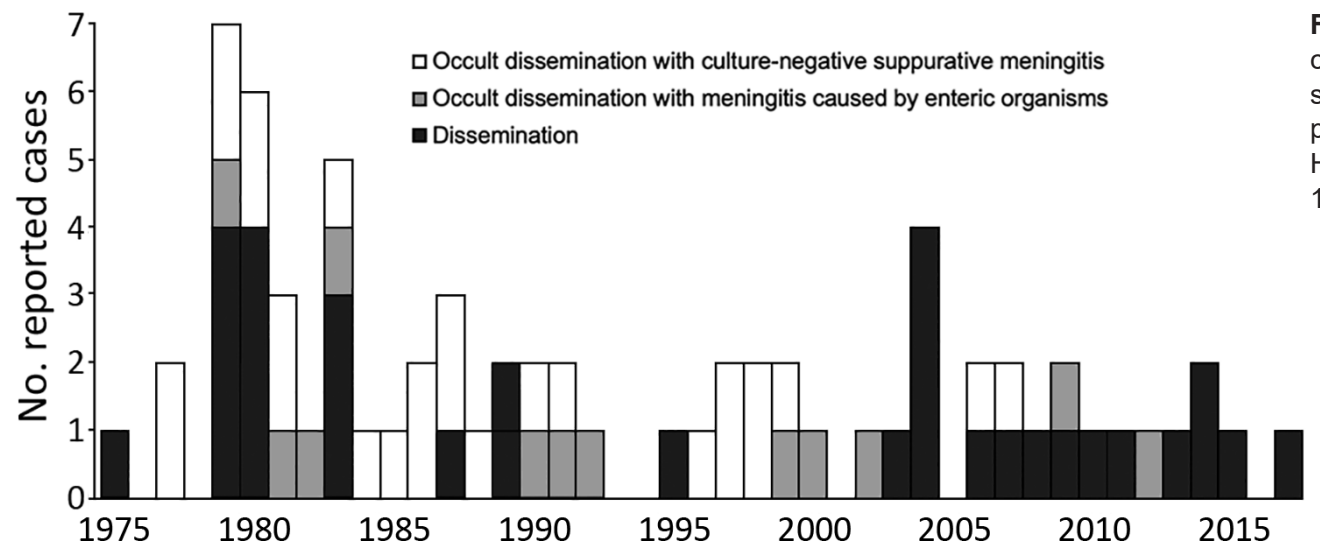

Figure. Number of reported cases of disseminated strongyloidiasis, by clinical phenotype, Okinawa Chubu Hospital, Uruma, Japan, 1975-2017. 
Table 2. Symptoms and clinical diagnoses of patients with disseminated strongyloidiasis, Okinawa Chubu Hospital, Uruma, Japan, 1975-2017

\begin{tabular}{|c|c|c|c|c|}
\hline \multirow[b]{2}{*}{ Characteristic } & \multicolumn{4}{|c|}{ No. patients } \\
\hline & All patients & Dissemination & $\begin{array}{c}\text { Occult dissemination } \\
\text { with meningitis/enteric } \\
\text { organisms }\end{array}$ & $\begin{array}{l}\text { Occult dissemination } \\
\text { with culture-negative } \\
\text { suppurative meningitis }\end{array}$ \\
\hline No. patients & $70(100)$ & $32(45.7)$ & $12(17.1)$ & $26(37.1)$ \\
\hline \multicolumn{5}{|l|}{ Symptoms* } \\
\hline Fever & 37 (52.9) & $15(46.9)$ & $8(66.7)$ & $14(53.8)$ \\
\hline Headache & $23(32.9)$ & $3(9.4)$ & $7(58.3)$ & $13(50.0)$ \\
\hline Altered mental status & $17(24.3)$ & 7 (21.9) & $3(25.0)$ & $7(26.9)$ \\
\hline Nausea or vomiting & $10(14.3)$ & $4(12.5)$ & $1(8.3)$ & $5(19.2)$ \\
\hline Abdominal pain & $6(8.6)$ & $2(6.3)$ & $2(16.7)$ & $2(7.7)$ \\
\hline Anorexia & $5(7.1)$ & $4(12.5)$ & $1(8.3)$ & $0(0)$ \\
\hline Constipation & $1(1.4)$ & $0(0)$ & $1(8.3)$ & $0(0)$ \\
\hline Diarrhea & $1(1.4)$ & $0(0)$ & $0(0)$ & $1(3.8)$ \\
\hline \multicolumn{5}{|l|}{ Clinical diagnosis $†$} \\
\hline Full-blown dissemination $\ddagger$ & $9(12.9)$ & $9(28.1)$ & $0(0)$ & $0(0)$ \\
\hline Sepsis & $23(32.9)$ & $13(40.6)$ & $7(58.3)$ & $3(11.5)$ \\
\hline Meningitis (enteric) & $22(31.4)$ & $10(31.3)$ & $12(100)$ & $0(0)$ \\
\hline Meningitis (culture-negative) & $34(48.6)$ & $8(25)$ & $0(0)$ & $26(100)$ \\
\hline Pneumonia & $27(38.6)$ & $26(81.3)$ & $0(0)$ & $1(3.8)$ \\
\hline Paralytic ileus & $17(24.3)$ & $15(46.9)$ & $1(8.3)$ & $1(3.8)$ \\
\hline \multicolumn{5}{|c|}{$\begin{array}{l}\text { *Other symptoms that were only noted in patients with dissemination included cough ( } 2 \text { cases), and rash, fatigue, convulsion, and syncope ( } 1 \text { case each) } \\
\text { †Other diagnoses that were only identified in patients with dissemination included peritonitis ( } 6 \text { cases), dermatitis }(5 \text { cases), and esophagitis ( } 1 \text { case). } \\
\text { †Full-blown dissemination is the severest form of dissemination and hyperinfection, referring to patients with paralytic ileus and pneumonia caused by } \\
\text { enteric organisms, as well as either sepsis or meningitis caused by enteric organisms. }\end{array}$} \\
\hline
\end{tabular}

One case-patient with full-blown dissemination, a woman in her 60s, had fever, vomiting, headache, and altered mental status. On arrival to the hospital, the patient was obtunded, and neck stiffness was noted. The results of lumbar puncture were suggestive of bacterial meningitis caused by gram-negative bacilli. On direct microscopic examination, multiple larvae were identified in feces, sputum, CSF, ascites, and gastric juice. The patient's condition deteriorated despite treatment for both strongyloidiasis and bacterial meningitis. Her clinical course also was complicated by paralytic ileus and bacterial pneumonia caused by K. pneumoniae. Gram staining of CSF was notable for gram-negative bacilli, and both blood and CSF cultures yielded E. coli. The patient died on hospital day 11. On autopsy, larvae were identified in the skin and lungs (11).

The clinical course of patients with occult dissemination with bacterial meningitis caused by enteric organisms was less severe than full-blown dissemination, yet remained critical without appropriate treatment. One such patient, a man in his 50 s, sought care at our emergency department in the 1980s for a 1-day history of headache, vomiting, shaking, and chills. On examination, the patient had neck stiffness. Results of lumbar puncture were notable for a white blood cell count of 4,104 cells $/ \mu \mathrm{L}$ ( $77 \%$ of polymorphonuclear neutrophils). Larvae were detected in feces. The patient was started on thiabendazole, chloramphenicol, and ampicillin. Gram staining of CSF samples was negative, but culture later yielded
E. coli. Blood culture obtained on admission was negative. The patient responded well to the treatment, and larvae were no longer detected in feces by hospital day 6 (14).

Occult dissemination with culture-negative suppurative meningitis occurred in less critical conditions. One case-patient, a woman in her 90s, was brought to our hospital in the 1980s for altered mental status and chills. The patient was obtunded, and results of CSF analysis was consistent with suppurative meningitis (white blood cell count of 3,812/cells/ $\mu \mathrm{L}$ [73\% of polymorphonuclear neutrophils]). Gram staining of CSF was negative for organisms, and culture results were sterile. Parasitologic examination was positive only in feces. The patient responded well to antibiotics and thiabendazole and was discharged to home on hospital day 9 (15).

\section{Parasitologic and Microbiologic Results}

Larvae were identified from samples including stool (from 60/70 [85.7\%] patients), sputum (30/70 $[42.9 \%])$, gastric juice $(24 / 70[34.3 \%])$, ascites $(4 / 70$ $[5.7 \%])$, urine $(3 / 70[4.3 \%]), \operatorname{CSF}(2 / 70[2.9 \%])$, and biopsy specimens (Table 3). No antibody testing or PCR was used in making a diagnosis, except for 1 patient who had necrotizing esophagitis (23).

E. coli, K. pneumoniae, and S. gallolyticus were the most common organisms detected from blood and CSF cultures, causing $7 \%-10 \%$ of bacteremia. Other organisms that caused bacteremia included E. aerogenes, S. infantarius, and Enterococcus spp. 
Table 3. Parasitologic investigation and culture results of patients with disseminated strongyloidiasis, Okinawa Chubu Hospital, Uruma, Japan, 1975-2017*

\begin{tabular}{|c|c|c|c|c|}
\hline \multirow[b]{2}{*}{ Characteristic } & \multicolumn{4}{|c|}{ No. (\%) patients } \\
\hline & All patients & Dissemination & $\begin{array}{l}\text { Occult dissemination } \\
\text { with meningitis/enteric } \\
\text { organisms }\end{array}$ & $\begin{array}{l}\text { Occult dissemination } \\
\text { with culture-negative } \\
\text { suppurative meningitis }\end{array}$ \\
\hline No. patients & $70(100)$ & $32(45.7)$ & $12(17.1)$ & $26(37.1)$ \\
\hline \multicolumn{5}{|l|}{ Specimens with larvae† } \\
\hline Stool & 60 (85.7) & $22(68.8)$ & $12(100)$ & $26(100)$ \\
\hline Sputum & $30(42.9)$ & 30 (93.8) & $0(0)$ & $0(0)$ \\
\hline Gastric juice & $24(34.3)$ & $16(50)$ & $3(25)$ & $5(19.2)$ \\
\hline \multicolumn{5}{|l|}{ Blood culture results $\ddagger$} \\
\hline Escherichia coli & $6(8.6)$ & $4(12.5)$ & $2(16.7)$ & $0(0)$ \\
\hline Klebsiella pneumoniae & $7(10.0)$ & $4(12.5)$ & $1(8.3)$ & $2(7.7)$ \\
\hline Streptococcus gallolyticus & $5(7.1)$ & $1(3.1)$ & $3(25)$ & $1(3.8)$ \\
\hline \multicolumn{5}{|l|}{ CSF culture results $\ddagger$} \\
\hline E. coli & $9 / 65(13.8)$ & 4/27 (14.8) & $5(41.7)$ & $0(0)$ \\
\hline K. pneumoniae & $6 / 65(9.2)$ & $3 / 27(11.1)$ & $3(25)$ & $0(0)$ \\
\hline S. gallolyticus & $5 / 65(7.7)$ & $1 / 27(3.7)^{\prime}$ & $4(33.3)$ & $0(0)$ \\
\hline \multicolumn{5}{|c|}{$\begin{array}{l}{ }^{*} \text { CSF, cerebrospinal fluid. } \\
\text { †ln dissemination, larvae also were detected from ascites ( } 4 \text { cases), urine ( } 3 \text { cases), CSF ( } 2 \text { cases), and skin, bronchoalveolar lavage, and biopsies of the } \\
\text { lung, duodenum, and liver ( } 1 \text { case each). } \\
\text { fln dissemination, blood cultures also were positive for Enterobacter aerogenes, Enterococcus spp., and S. infantarius ( } 1 \text { case each), and CSF cultures } \\
\text { were positive for E. aerogenes and Streptococcus spp. ( } 1 \text { case each). In occult dissemination with meningitis (enteric organisms), blood cultures also } \\
\text { were positive for E. agglomerans ( } 1 \text { case), and CSF cultures were positive for Enterococcus spp. ( } 1 \text { case). }\end{array}$} \\
\hline
\end{tabular}

\section{Treatment}

Before 2002, patients were treated with thiabendazole. Thiabendazole was administered at the conventional dose of $50 \mathrm{mg} / \mathrm{kg} /$ day (not exceeding 3 $\mathrm{g} / \mathrm{d}$ ) for $\geq 3$ days. Patients in critical conditions were given double doses $(100 \mathrm{mg} / \mathrm{kg} / \mathrm{d})$ for the first $1-2$ days. Thiabendazole was tapered off when clinical improvement or disappearance of larvae in feces was confirmed.

After 2003, all patients were treated with ivermectin at the dose of $200 \mu \mathrm{g} / \mathrm{kg} /$ day. Two patients with critical conditions received 2 or 1.5 times the conventional dose. Ivermectin was administered either 1-2 weeks apart or consecutively until the confirmation of clinical improvement or disappearance of larvae from feces. A combination of oral and rectal forms of ivermectin was administered in 3 patients.

One patient in 1980 initially received pyrvinium pamoate at a dose of $20 \mathrm{~mL} /$ day because thiabendazole was not available. Another patient with occult dissemination with culture-negative suppurative meningitis did not receive anthelmintics because meningitis was suspected to be caused by the central nervous system involvement of adult T-cell leukemia.

\section{Discussion}

Disseminated strongyloidiasis is a medical emergency. Because of its rarity, most previous reports consist of single cases (24), making it difficult to determine its clinical phenotypes. Previous reports identified atypical suppurative meningitis, defined as communityacquired meningitis caused by enteric organisms or culture-negative suppurative meningitis, as a form of occult dissemination $(10,14-16)$. Our study corroborates preceding analyses and extends their findings by demonstrating that early recognition of occult dissemination is lifesaving.

Demographic characteristics in our study, such as age distribution and male-to-female ratio, were in keeping with previous reports (24). However, our patients all were born before 1964, and the number of annually reported cases was decreasing during the study period. These findings were consistent with a report from another institution in Okinawa (25) and suggest that patients, predominantly men, in this region in the past became infected from contaminated soil during farming and by walking barefoot (26). Improved farming environments and changes in lifestyle might have reduced the risk for exposure. Another trend we noted was that 9 of 11 reported cases during the last 10 years of the study period were classified as dissemination and only 2 out of 11 as occult dissemination. The median age of patients in these 11 cases was 80 years, implying that the elderly who had been exposed to $S$. stercoralis parasites in their youth developed systemic strongyloidiasis at advanced age, which quickly disseminated because of their impaired immune status.

In this case series, few patients had risk factors for strongyloidiasis other than HTLV-1 infection, including recent steroid use (3), organ transplant $(4)$, or cirrhosis $(27,28)$. In areas with HTLV-1 endemicity, HTLV-1 infection is the major risk factor for both chronic strongyloidiasis and dissemination (29). 
Table 4. Involvement of larvae and enteric organisms in disseminated strongyloidiasis reported in previous studies*

\begin{tabular}{lccc}
\hline Characteristic & Dissemination & $\begin{array}{c}\text { Occult dissemination with } \\
\text { meningitis/enteric } \\
\text { organisms }\end{array}$ & $\begin{array}{c}\text { Occult dissemination with } \\
\text { culture-negative } \\
\text { suppurative meningitis }\end{array}$ \\
\hline Involvement of larvae & $\begin{array}{c}\text { Confirmed (rhabditiform and } \\
\text { filariform larvae) }\end{array}$ & $\begin{array}{c}\text { Confirmed (rhabditiform } \\
\text { larvae) }\end{array}$ & $\begin{array}{c}\text { Confirmed (rhabditiform } \\
\text { larvae) }\end{array}$ \\
\hline Involvement of enteric organisms & Confirmed & Confirmed & Not detected \\
\hline${ }^{*}$ References 11, 14, and 15. & & & \\
\hline
\end{tabular}

Although diabetes mellitus has been reported to be a risk factor for strongyloidiasis treatment failure (30), the prevalence in our series remained low.

Considering the mortality rates and the various symptoms and clinical diagnoses, dissemination is the most severe form of strongyloidiasis, followed by occult dissemination with meningitis caused by enteric organisms and then occult dissemination with culture-negative suppurative meningitis. Evidence of multiorgan involvement was less common in occult dissemination, which might reflect the degree of involvement of larvae and enteric organisms in these 3 phenotypes. Previous studies have identified different degrees of their involvement $(11,14,15)$ (Table 4).

Despite the low mortality rate associated with occult dissemination with culture-negative suppurative meningitis, it is critical for clinicians to identify concomitant strongyloidiasis because patients might later have recurrent meningitis in more critical conditions (18) if not properly treated. Culture-negative suppurative meningitis should prompt the immediate consideration of performing a parasitologic examination in areas where strongyloidiasis and HTLV-1 are endemic. Previous reports have suggested that culture-negative suppurative meningitis is a form of dissemination $(10,15,17)$.

Limited data are available about the optimal treatment of dissemination. Treatment options consist of reducing immunosuppressive therapies and initiating antihelmintics and antibiotics. Reducing immunosuppressive therapies is recommended because immunosuppressive therapies can induce dissemination (6). In a systematic review comparing ivermectin, albendazole, and thiabendazole, ivermectin resulted in better parasitologic cure than albendazole and fewer adverse effects than thiabendazole (31). The conventional dose of ivermectin is $200 \mu \mathrm{g} / \mathrm{kg} /$ day orally for 2 days, either consecutively or 2 weeks apart (32). In dissemination, the dose and duration are determined clinically. Repeated or prolonged administration are preferred until patients respond or until larvae are no longer detected (33). For patients who are unable to take oral ivermectin, a rectal (34), veterinary subcutaneous (35), or parenteral (36) formula is administered. The use of veterinary ivermectin is considered in critical cases. Some studies have also reported the use of a combination of ivermectin and albendazole (37). In our study, several critical patients received an increased dose of thiabendazole or ivermectin. No patients received a combination of ivermectin and thiabendazole. Anthelmintic agents were continued until patients responded or until larvae disappeared.

Initiation of antibiotics that target enteric organisms is essential. The antibiotics must penetrate into the central nervous system when meningitis is suspected. The spectrum should be based on the local antibiogram. In our study, no patients had bacterial infection that produced extended spectrum $\beta$-lactamases or AmpC $\beta$-lactamases. Clinicians should also notice that the results of blood and CSF cultures differed in 4 patients (4/70 [5.7\%]), suggesting that larvae might have carried multiple enteric organisms. Empiric antibiotics that sufficiently cover most enteric organisms, such as third-generation cephalosporins, are recommended. The administration of dexamethasone for bacterial meningitis should carefully be considered because it might induce dissemination (9).

Our investigation has several limitations. First, the retrospective nature of this study might have resulted in distortions in the accuracy of the retrieved information. Second, patients with disseminated strongyloidiasis have also been admitted to other medical centers in Okinawa (38); hence, we might have underreported the prevalence of dissemination in this region. Third, the findings cannot be generalizable to regions where HTLV-1 infection is not endemic.

In summary, we classified disseminated strongyloidiasis into 3 phenotypes on the basis of their severity and various symptoms: dissemination, occult dissemination with meningitis caused by enteric organisms, and occult dissemination with culture-negative suppurative meningitis. Early identification of occult dissemination is lifesaving. Treatment options consist of reduction of immunosuppressive therapies, initiation of anthelmintic agents with a higher dose and longer duration depending on the severity of symptoms, and concurrent use of antimicrobial agents against enteric organisms. 


\section{Acknowledgment}

We thank Kiyofumi Ohkusu for conducting real-time PCR analysis.

\section{About the Author}

Dr. Mukaigawara was a chief medical resident at Okinawa Chubu Hospital when he conducted this research. He is now a graduate student in public policy at Harvard Kennedy School. His primary research interests include global health governance and socioeconomic determinants of health.

\section{References}

1. Olsen A, van Lieshout L, Marti H, Polderman T, Polman K, Steinmann P, et al. Strongyloidiasis - the most neglected of the neglected tropical diseases? Trans R Soc Trop Med Hyg. 2009;103:967-72. https:/ / doi.org/10.1016/ j.trstmh.2009.02.013

2. Igra-Siegman Y, Kapila R, Sen P, Kaminski ZC, Louria DB. Syndrome of hyperinfection with Strongyloides stercoralis. Rev Infect Dis. 1981;3:397-407. https://doi.org/10.1093/ clinids/3.3.397

3. Fardet L, Généreau T, Poirot JL, Guidet B, Kettaneh A, Cabane J. Severe strongyloidiasis in corticosteroidtreated patients: case series and literature review. J Infect. 2007;54:18-27. https://doi.org/10.1016/j.jinf.2006.01.016

4. Marty FM. Strongyloides hyperinfection syndrome and transplantation: a preventable, frequently fatal infection. Transpl Infect Dis. 2009;11:97-9. https:/ / doi.org/10.1111/ j.1399-3062.2009.00383.x

5. Sánchez PR, Guzman AP, Guillen SM, Adell RI, Estruch AM, Gonzalo IN, et al. Endemic strongyloidiasis on the Spanish Mediterranean coast. QJM. 2001;94:357-63. https://doi.org/10.1093/qjmed/94.7.357

6. Keiser PB, Nutman TB. Strongyloides stercoralis in the immunocompromised population. Clin Microbiol Rev. 2004;17:208-17. https://doi.org/10.1128/CMR.17.1. 208-217.2004

7. US Centers for Disease Control and Prevention. Parasites: Strongyloides. Biology [cited 2019 Feb 1]. https:/ / www.cdc.gov/ parasites/strongyloides/biology.html

8. Mansfield LS, Alavi A, Wortman JA, Schad GA. Gamma camera scintigraphy for direct visualization of larval migration in Strongyloides stercoralis-infected dogs. Am J Trop Med Hyg. 1995;52:236-40. https:/ / doi.org/10.4269/ ajtmh.1995.52.236

9. Pukkila-Worley R, Nardi V, Branda JA. Case records of the Massachusetts General Hospital. Case 28-2014. A 39-year-old man with a rash, headache, fever, nausea, and photophobia. N Engl J Med. 2014;371:1051-60. https:/ / doi.org/10.1056/ NEJMcpc1405886

10. Kishaba T. Disseminated strongyloidiasis. In: Shiroma Y, Sato Y, editors. Strongyloides stercoralis and strongyloidiasis in Japan [in Japanese]. Fukuoka (Japan): Kyushu University Press; 1997. p. 55-78.

11. Kishaba T. Strongyloidiasis and infections due to enteric organisms: sepsis, pneumonia and meningitis caused by Gram-negative bacteria associated with disseminated strongyloidiasis [in Japanese]. Infection. 1982;12:180.

12. Jain AK, Agarwal SK, el-Sadr W. Streptococcus bovis bacteremia and meningitis associated with Strongyloides stercoralis colitis in a patient infected with human immunodeficiency virus. Clin Infect Dis. 1994;18:253-4. https://doi.org/10.1093/clinids/18.2.253

13. Chu E, Whitlock WL, Dietrich RA. Pulmonary hyperinfection syndrome with Strongyloides stercoralis. Chest. 1990;97: 1475-7. https://doi.org/10.1378/chest.97.6.1475

14. Kishaba T, Uchihara T, Ueno K, Goeku C, Shimabukuro Y. Gram-negative rod meningitis probably caused by occult disseminated strongyloidiasis [in Japanese]. Okinawa Medical Journal. 1985;22:3.

15. Kishaba T, Suginohara K, Tamaki K, Miyara Y, Endo K, Taira $Y$, et al. Culture negative suppurative meningitis probably caused by occult disseminated strongyloidiasis [in Japanese]. Okinawa Medical Journal. 1989;26:219.

16. Mukaigawara M, Nakayama I, Gibo K. Strongyloidiasis and culture-negative suppurative meningitis, Japan, 1993-2015. Emerg Infect Dis. 2018;24:2378-80. https:/ / doi.org/10.3201/ eid2412.180375

17. Sasaki Y, Taniguchi T, Kinjo M, McGill RL, McGill AT, Tsuha S, et al. Meningitis associated with strongyloidiasis in an area endemic for strongyloidiasis and human T-lymphotropic virus-1: a single-center experience in Japan between 1990 and 2010. Infection. 2013;41:1189-93. https:/ / doi.org/10.1007/s15010-013-0483-2

18. Shimasaki T, Chung H, Shiiki S. Five cases of recurrent meningitis associated with chronic strongyloidiasis. Am J Trop Med Hyg. 2015;92:601-4. https:/ / doi.org/10.4269/ ajtmh.14-0564

19. Toma H, Shimabukuro I, Kobayashi J, Tasaki T, Takara M, Sato Y. Community control studies on Strongyloides infection in a model island of Okinawa, Japan. Southeast Asian J Trop Med Public Health. 2000;31:383-7.

20. Sagara Y, Iwanaga M, Morita M, Sagara Y, Nakamura H, Hirayama $\mathrm{H}$, et al. Fine-scale geographic clustering pattern of human T-cell leukemia virus type 1 infection among blood donors in Kyushu-Okinawa, Japan. J Med Virol. 2018;90:1658-65. https://doi.org/10.1002/jmv.25239

21. Cresci GA, Bawden E. Gut microbiome: what we do and don't know. Nutr Clin Pract. 2015;30:734-46. https:/ / doi.org/ $10.1177 / 0884533615609899$

22. Scowden EB, Schaffner W, Stone WJ. Overwhelming strongyloidiasis: an unappreciated opportunistic infection. Medicine (Baltimore). 1978;57:527-44. https:/ / doi.org/10.1097/ 00005792-197811000-00004

23. Tomori M, Mukaigawara M, Narita M. Acute esophageal necrosis associated with Strongyloides stercoralis hyperinfection. Am J Trop Med Hyg. 2019;100:1037-8. https:/ / doi.org/ 10.4269/ajtmh.18-0664

24. Geri G, Rabbat A, Mayaux J, Zafrani L, Chalumeau-Lemoine L, Guidet B, et al. Strongyloides stercoralis hyperinfection syndrome: a case series and a review of the literature. Infection. 2015;43:691-8. https:/ / doi.org/10.1007/s15010-015-0799-1

25. Tanaka T, Hirata T, Parrott G, Higashiarakawa M, Kinjo T, Kinjo T, et al. Relationship among Strongyloides stercoralis infection, human T-cell lymphotropic virus type 1 infection, and cancer: a 24-year cohort inpatient study in Okinawa, Japan. Am J Trop Med Hyg. 2016;94:365-70. https:/ / doi.org/ 10.4269/ajtmh.15-0556

26. Schär F, Trostdorf U, Giardina F, Khieu V, Muth S, Marti $\mathrm{H}$, et al. Strongyloides stercoralis: global distribution and risk factors. PLoS Negl Trop Dis. 2013;7:e2288. https:/ / doi. org/10.1371/journal.pntd.0002288

27. Teixeira MC, Pacheco FT, Souza JN, Silva ML, Inês EJ, Soares NM. Strongyloides stercoralis infection in alcoholic patients. BioMed Res Int. 2016;2016:4872473. https:/ / doi.org/ $10.1155 / 2016 / 4872473$ 
28. de Oliveira LC, Ribeiro CT, Mendes DM, Oliveira TC, Costa-Cruz JM. Frequency of Strongyloides stercoralis infection in alcoholics. Mem Inst Oswaldo Cruz. 2002;97:119_ 21. https://doi.org/10.1590/S0074-02762002000100021

29. Gotuzzo E, Terashima A, Alvarez H, Tello R, Infante R, Watts DM, et al. Strongyloides stercoralis hyperinfection associated with human T cell lymphotropic virus type-1 infection in Peru. Am J Trop Med Hyg. 1999;60:146-9. https://doi.org/10.4269/ajtmh.1999.60.146

30. Hays R, Esterman A, McDermott R. Type 2 diabetes mellitus is associated with Strongyloides stercoralis treatment failure in Australian aboriginals. PLoS Negl Trop Dis. 2015;9:e0003976. https:/ / doi.org/10.1371/journal.pntd.0003976

31. Henriquez-Camacho C, Gotuzzo E, Echevarria J, White AC Jr, Terashima A, Samalvides F, et al. Ivermectin versus albendazole or thiabendazole for Strongyloides stercoralis infection. Cochrane Database Syst Rev. 2016;(1):CD007745. https://doi.org/10.1002/14651858.CD007745.pub3

32. Zaha O, Hirata T, Kinjo F, Saito A, Fukuhara H. Efficacy of ivermectin for chronic strongyloidiasis: two single doses given 2 weeks apart. J Infect Chemother. 2002;8:94-8. https:/ / doi.org/10.1007/s101560200013

33. Mejia R, Nutman TB. Screening, prevention, and treatment for hyperinfection syndrome and disseminated infections caused by Strongyloides stercoralis. Curr Opin Infect Dis. 2012; 25:458-63. https:// doi.org/10.1097/QCO.0b013e3283551dbd

34. Tarr PE, Miele PS, Peregoy KS, Smith MA, Neva FA, Lucey DR. Case report: Rectal adminstration of ivermectin to a patient with Strongyloides hyperinfection syndrome. Am J Trop Med Hyg. 2003;68:453-5. https://doi.org/10.4269/ ajtmh.2003.68.453

35. Barrett J, Broderick C, Soulsby H, Wade P, Newsholme W. Subcutaneous ivermectin use in the treatment of severe Strongyloides stercoralis infection: two case reports and a discussion of the literature. J Antimicrob Chemother. 2016;71:220-5. https://doi.org/10.1093/jac/dkv315

36. Marty FM, Lowry CM, Rodriguez M, Milner DA, Pieciak WS, Sinha A, et al. Treatment of human disseminated strongyloidiasis with a parenteral veterinary formulation of ivermectin. Clin Infect Dis. 2005;41:e5-8. https:/ / doi.org/ 10.1086/430827

37. Pornsuriyasak P, Niticharoenpong K, Sakapibunnan A. Disseminated strongyloidiasis successfully treated with extended duration ivermectin combined with albendazole: a case report of intractable strongyloidiasis. Southeast Asian J Trop Med Public Health. 2004;35:531-4.

38. Kishimoto K, Hokama A, Hirata T, Ihama Y, Nakamoto M, Kinjo N, et al. Endoscopic and histopathological study on the duodenum of Strongyloides stercoralis hyperinfection. World J Gastroenterol. 2008;14:1768-73. https:/ / doi.org/10.3748/ wjg. 14.1768

Address for correspondence: Mitsuru Mukaigawara, Harvard Kennedy School, 79 John F. Kennedy St, Cambridge, MA 02138 USA; email: mitsuru_mukaigawara@hks.harvard.edu

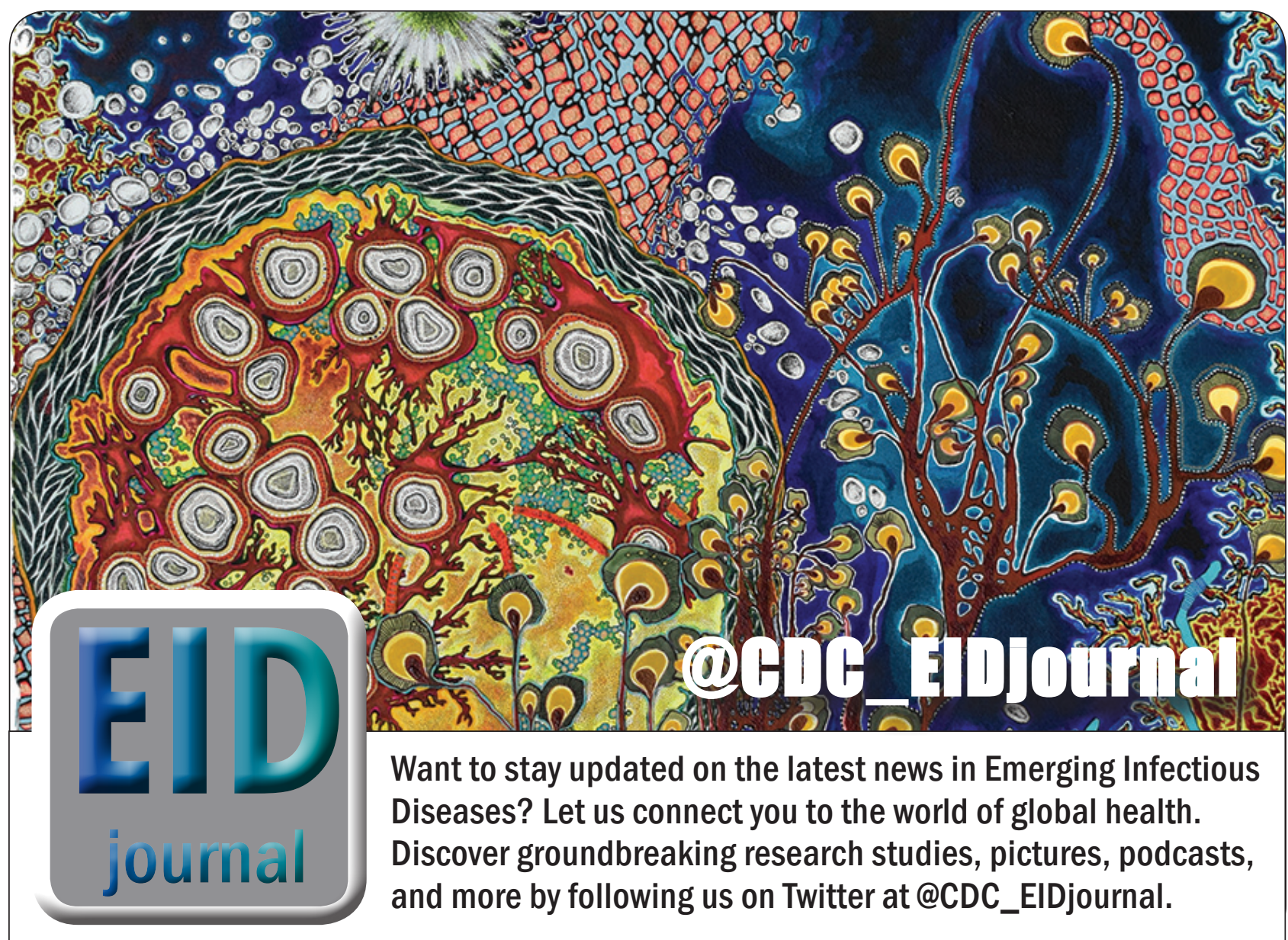

